\title{
Evaluation of bone marrow mononuclear cells as an adjunct therapy to minced muscle graft for the treatment of volumetric muscle loss injuries
}

\author{
Stephen M. Goldman, Beth E. P. Henderson and Benjamin T. Corona*
}

\begin{abstract}
Background: The delivery of alternative myogenic cell sources to enhance the efficacy of minced muscle grafts (MG) for the treatment of volumetric muscle loss (VML) injuries is a promising strategy to overcome the demand on muscle-derived donor tissue that currently limits the translation of this therapy.

Methods: Using a rat model of VML, bone marrow mononuclear cells (BMNCs) were evaluated for their ability to directly contribute to de novo muscle fiber regeneration by transplanting MG in a collagen carrier at a dose of 50\% of the VML injury both with and without concomitant delivery of 5 million BMNCs derived via density gradient centrifugation from the bone marrow of a syngeneic green fluorescent protein (GFP) $)^{+}$donor.

Results: Histological, molecular, and functional analyses revealed that BMNCs can engraft with co-delivered MG and contribute to nascent myofiber, but do so at a low magnitude without resulting in significant changes to transcription of key myogenic genes or gains in whole muscle force generation relative to MG alone.

Conclusion: As such, co-delivery of BMNCs with MG is a promising treatment paradigm to VML that will require further investigation to identify the phenotype and therapeutic dosing of the bone marrow-derived cell populations which engraft most efficiently.
\end{abstract}

Keywords: Bone marrow mononuclear cells, Volumetric muscle loss, Skeletal muscle, Regenerative medicine, Minced muscle graft, Musculoskeletal trauma

\section{Background}

Volumetric muscle loss (VML) is a condition characterized by the loss of tissue beyond the endogenous regenerative capacity of the affected musculature and the associated persistent functional deficit for which there is no current standard of care [1]. Transplantation of minced muscle grafts (MG) is a potential therapy for some VML indications that has been shown to be rich in pax $7^{+}$satellite cells and effective in promoting meaningful de novo fiber regeneration at doses $\geq 50 \%$ of the tissue lost due to injury [2-4]. Furthermore, MG falls under the US Food \& Drug Administration's definition

\footnotetext{
* Correspondence: benjamin.t.corona.civ@mail.mil; Corona.benjamin.t@gmail.com

United States Army Institute of Surgical Research, 3698 Chambers Pass, Bldg. 3611, Fort Sam Houston, TX 78234, USA
}

of a minimally manipulated tissue, and thus represents a near-term solution. Clinically, the demands on muscle autograft become a limiting factor for cases presenting large or multiple injuries. While pax $7^{+}$satellite cells are an absolute requirement for de novo myofiber regeneration [5], alternative cell types are capable of contributing to nascent myofibers in the presence of satellite cells [6-9]. As such, delivery of nonmuscle-derived autologous stem cells shows promise as an adjunct therapy to enhance the regenerative capacity of MG, thereby diminishing sourcing limitations and raising the possibility of treating larger VML injuries.

Bone marrow is a rich source of stem cells and has a well-developed history in transplant medicine. Bone marrow-derived cells are expected to promote healing by multiple effector functions including the production 
of growth factors, pro-inflammatory cytokines, and antiinflammatory cytokines which stimulate cell proliferation, inhibit apoptosis, recruit cells, reduce fibrosis, and induce angiogenesis [10, 11]. Moreover, unfractionated bone marrow cells have been shown to participate in muscle regeneration in a recoverable cardiotoxin-induced injury model [12], and to enhance minced graft-mediated myogenesis in coculture implants in the peritoneal cavity of mice, quadriceps in the rat, and monkey arm VML injuries [13-16]. While these results are promising, further fractionation of bone marrow cells to isolate the mononuclear fraction, consisting of B cells $(\sim 50 \%)$, monocytes/dendritic cells $(\sim 5 \%)$, other $\mathrm{CD} 45^{+}$cells (e.g., $\mathrm{CD} 34^{+} \mathrm{CD} 105^{-}$hematopoeitic stem cells) and a variety of $\mathrm{CD} 45^{-}$cells (e.g., $\mathrm{CD} 45^{-} \mathrm{CD} 105^{+}$mesenchymal stem cells), is expected to result in greater myogenic efficacy when delivered in combination with a MG therapy [17]. This expectation is based on the fact that delivery of the bone marrow mononuclear cell (BMNC) fraction enriches the therapy for the resident stem cell populations of the bone marrow and removes red blood cells and platelets which might deleteriously impact the efficacy of said stem cells [18]. Furthermore, BMNCs have also been shown to improve skeletal muscle function, as case reports have highlighted improved muscle function following BMNC transplantation in children with Duchenne muscular dystrophy $[19,20]$. It is for these reasons, and the fact that separation of the bone marrow into various cell fractions is clinically mature and relatively simple to perform, that we sought to evaluate BMNCs as an adjunct therapy to MG.

In this study, we used a rat model of VML to test the hypothesis that BMNCs directly contribute to de novo muscle fiber regeneration by transplanting MG in a collagen carrier at a dose of $50 \%$ of the VML injury both with and without concomitant delivery of 5 million BMNCs derived via density gradient centrifugation from the bone marrow of a syngeneic green fluorescent protein (GFP) expressing donor (Fig. 1). Biochemical and immunofluorescence analyses were performed at 2 and 8 weeks with in vivo assessment of muscle function at 8 weeks.

\section{Materials and methods VML model}

Under anesthesia (1-3\% isoflurane) and in sterile conditions, a lateral incision was made through the skin lengthwise along the lateral aspect of the tibialis anterior (TA) muscle of the left hind limb of adult male Lewis rats (Harlan Laboratories, Indianapolis, IN, USA). After

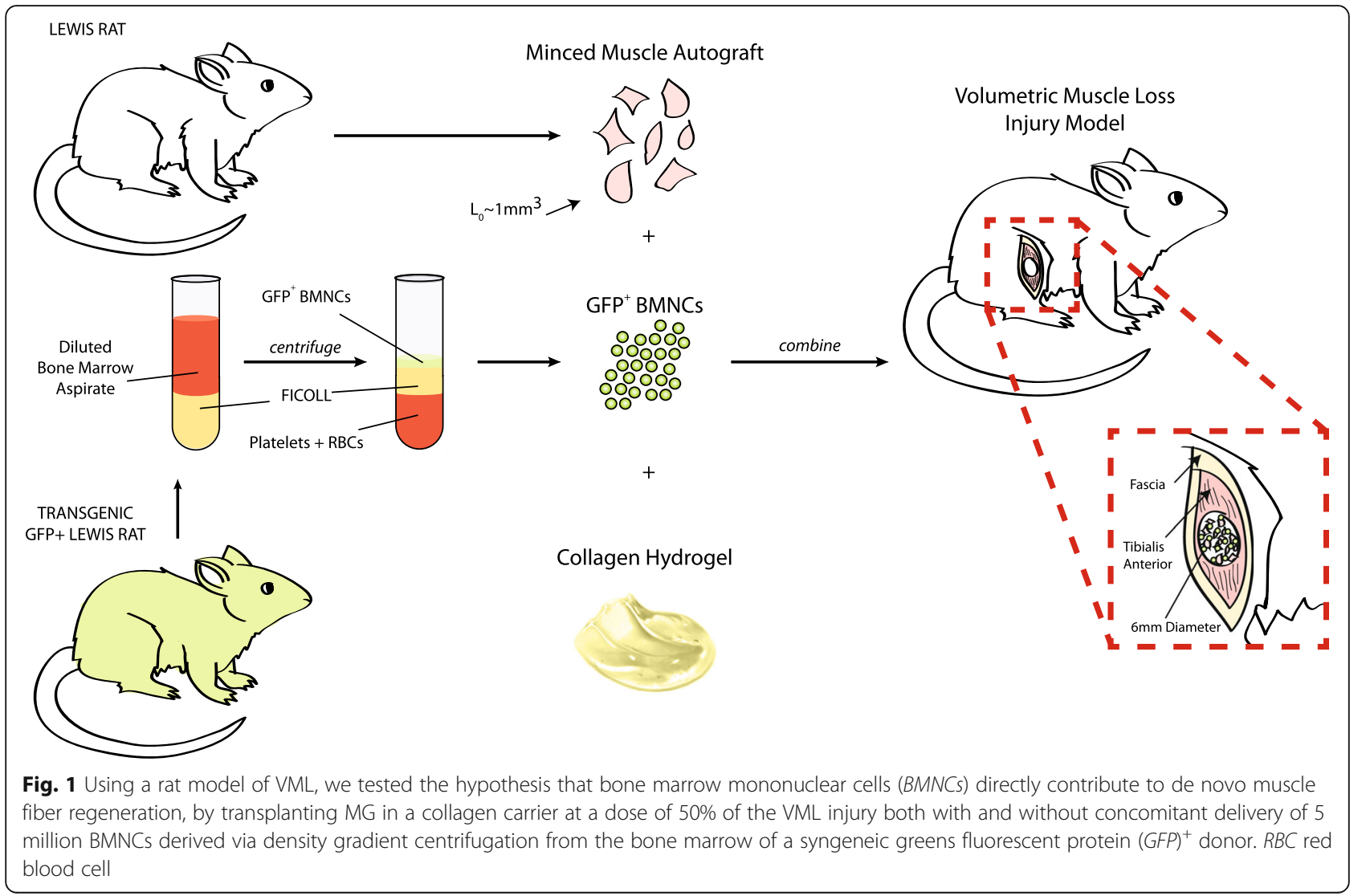


reflecting the skin and fascia from the anterior surface, the TA muscle and underlying extensor digitorum longus (EDL) muscle were separated by blunt dissection. A metal plate was inserted between the muscles and a 6-mm biopsy punch was used to excise a $\sim 20 \%$ volume defect, and MG constructs were delivered acutely to the VML wound bed. The wound was closed in layers by suturing (fascia) and stapling (skin).

\section{BMNC isolation}

Bone marrow was flushed from the intramedullary space of the long bones from transgenic GFP-expressing Lewis rats (Lew-Tg(CAG-EGFP), Rat Resource \& Research Center, Columbia, MO, USA), and diluted to a total volume of $4 \mathrm{~mL}$ with Hank's Balanced Salt Solution. BMNCs were isolated from the diluted bone marrow aspirate on a Ficoll $(3 \mathrm{~mL})$ density gradient (Ficoll-Paque ${ }^{\mathrm{Tx}}$ PLUS, $1.077 \mathrm{~g} / \mathrm{mL}$; GE Healthcare, Buckinghamshire, UK) after unbraked centrifugation (400 g) at room temperature for $40 \mathrm{~min}$. After washing twice, viable BMNC yield was assessed via Trypan Blue exclusion and automated cell counter (Countess ${ }^{\mathrm{Th}}$, Invitrogen, Carlsbad, CA, USA).

\section{Construct preparation}

MG were derived from the TA muscle of a syngeneic wild-type Lewis rat. The excised muscle tissue was minced into $\sim 1 \mathrm{~mm}^{3}$ pieces. After mincing, MG at a dose equal to approximately $50 \%$ of the frank loss of tissue was mixed into a collagen $(400 \mu \mathrm{L}, 3 \mathrm{mg} / \mathrm{mL})$ solution with or without 5 million BMNCs (dose based on single donor BMNC yield) and cast within the wells of a 48-well cell culture plate. The collagen constructs were then allowed to crosslink at $37{ }^{\circ} \mathrm{C}$ for $30 \mathrm{~min}$, after which time the constructs were transplanted to the VML defect.

\section{In vivo functional assessments}

With the animal in a supine position, the knee of the rat was secured in place at $90^{\circ}$ by a clamp apparatus. The foot was strapped to a boot-like foot pedal with the ankle at a $90^{\circ}$ angle connected to a servomotor controlled force-displacement transducer (Aurora Scientific, Aurora, ON, USA). The peroneal nerve was stimulated using percutaneous needle electrodes placed around the peroneal nerve. Optimal voltage was determined using a series of twitch and tetanic contractions. Contractile function of the TA muscle was assessed by first severing the distal tendon of the synergist EDL muscle and measuring peak isometric torque as a function of stimulation frequency (400 ms train; $0.1 \mathrm{~ms}$ pulse width; $1-10 \mathrm{~V}$; 10-200 Hz). The servomotor input and force and displacement transducer outputs are controlled and acquired, respectively, using a $\mathrm{PC}$ equipped with a data acquisition board (National Instruments) and custom designed Lab View (National Instruments) based software program.

\section{Transcriptional analysis}

A portion of the defect region of the VML injury was collected at 2 and 8 weeks post-injury and immediately snap frozen in liquid nitrogen. Reverse transcription quantitative polymerase chain reaction (RT-qPCR) was used to quantify gene expression within the VML defect region. RNA was isolated from the homogenized tissue using the TRIzol method. The RNA samples were reverse transcribed into cDNA using a QuantiTech Rev Transcription kit (Qiagen, Hilden, Germany) according to the manufacturer's protocol. Gene expression for target markers was determined using custom-designed primers for embryonic myosin heavy chain (eMHC,

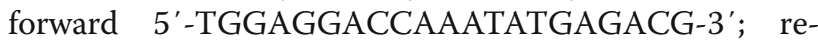
verse $5^{\prime}$-CACCATCAAGTCCTCCACCT-3') and myogenin (forward 5'-CTACAGGCCTTGCTCAGCTC-3'; reverse 5'-GTTGGGACCAAACTCCAGTG-3') with RT-qPCR amplification performed in the presence of SYBR Green (Bio-Rad Laboratories, Hercules, CA, USA). The raw fluorescence data were processed using LinRegPCR (v12.11; http://www.hartfaalcentrum.nl) with glyceraldehyde-3-phosphate dehydrogenase (GAPDH) serving as the endogenous control. Expression $(n=4-6$ per group) of each target gene was calculated relative to samples from the contralateral limb.

\section{Immunofluorescence analysis}

A portion of the neotissue from the defect region was embedded in a talcum-based gel, frozen in 2methylbutane, and supercooled in liquid nitrogen. Cryosections $(8 \mu \mathrm{m})$ were prepared and probed for laminin (1:100; catalog ab34360; Abcam) and GFP (1:100; catalog ab6673; Abcam) and detected with fluorescent antibodies (1:200; catalog A11055 and A21207; Invitrogen) to assess direct myogenic contribution of BMNCs. Qualitative assessments were made by observing three sections (separated by no less than $160 \mu \mathrm{m}$ ) from five muscles per group.

\section{Statistical analysis}

Data are reported as the mean \pm SEM with statistically significant differences defined as $p<0.05$ using two-way analysis of variance (ANOVA) with Tukey's post-hoc tests for multiple comparisons. Sample sizes for gene expression and in vivo neuromuscular strength assessments are $n=4-6$ per group and time point.

\section{Results}

A BMNC subpopulation definitively contributed to muscle fiber regeneration as evidenced by immunofluorescence 
staining of $\mathrm{GFP}^{+}$muscle fibers in the defect regions of VML-injured wild-type hosts (Fig. 2a). The overall direct contribution to myofiber regeneration, however, was low and localized to small regions within the broader defect area which was otherwise filled with what are assumed to be MG derived neofibers and extracellular matrix based on

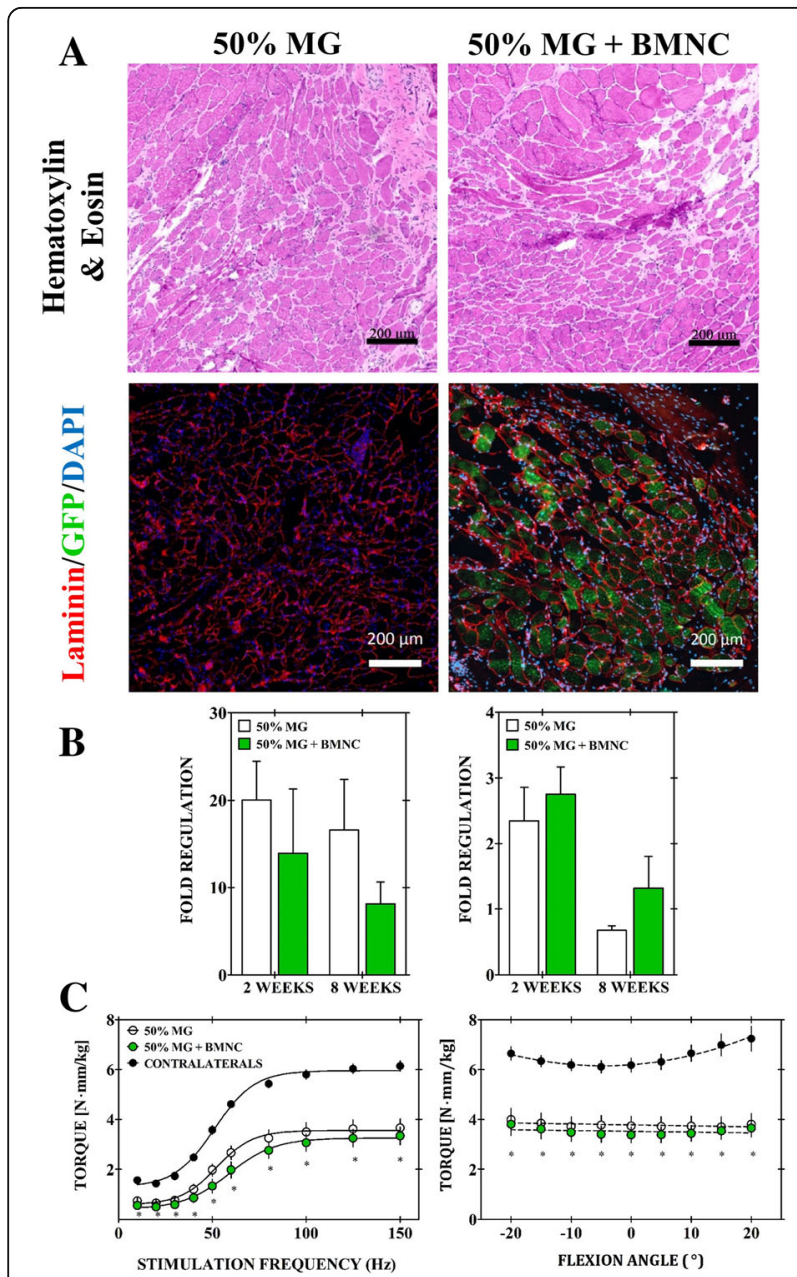

Fig. 2 Bone marrow mononuclear cells (BMNCS) definitively contribute to de novo muscle fiber regeneration, but do not promote transcriptional or functional gains beyond those provided by minced muscle graft (MG). a Representative hematoxylin and eosin sections and immunofluorescence staining of green fluorescent protein (GFP) ${ }^{+}$muscle fibers in the defect regions of $50 \%$ MG + BMNC (right) and 50\% MG treated VML injuries (left) in wild-type hosts at 8 weeks post-injury. $\mathbf{b}$ Transcription of myogenesis markers, embryonic myosin heavy chain (eMHC) and myogenin, was not significantly different between BMNC supplemented and unsupplemented MG repair groups for either of the time points investigated $(p>0.05)$. c BMNC contribution to myofiber regeneration did not result in a change in in vivo isometric tetanic force generation of treated TA muscles at 8 weeks post-injury for any of the flexion angles tested. Values are shown as means \pm SEM. ${ }^{*} p<0.05,50 \% \mathrm{MG}$ and $50 \% \mathrm{MG}+$ BMNC groups versus contralateral limb prior observations [3]. This observation was supported on a transcriptional level, as no significant difference was measured between the 50\% MG and 50\% MG with BMNC groups for the expression of the genes comprising our myogenic panel (eMHC, myogenin) at either of the time points investigated (Fig. 2b). Furthermore, BMNC contribution to muscle fiber regeneration does not result in an appreciable increase in the force generation of VMLinjured muscle. There was no observed difference in peak isometric torque between BMNC supplemented and MG repair groups at the 8-week time point (Fig. 2c). Forcefrequency curves for the two groups were statistically similar across all stimulation frequencies and joint angles with similar peak isometric torque deficits of $44.6 \pm 6.1 \%$ (MG) and $45.6 \pm 6.7 \%$ (MG + BMMC) relative to the unaffected contralateral control limbs.

\section{Discussion}

While the initial goal of identifying an autologous cell source requiring minimal manipulation prior to usage as an adjunct therapy to enhance the therapeutic effect of MG on VML was not achieved, the findings of this study provide strong justification for further pursuit of a bone marrow-derived stem cell source for supplementation of MG therapy to VML. Some portion of the heterogeneous BMNC population delivered herein contributed to myofiber regeneration in agreement with previous findings [12]. What was not determined, however, was which subgroups of the greater BMNC population are responsible for the observed engraftment. Studies by Sherwood et al. [8] and Polesskaya et al. [9] independently evaluated the myogenic capacity of these bone marrowderived cell types and produced conflicting reports with respect to capacity for myogenic differentiation of the non-hematopoietic $\left(\mathrm{CD} 45^{-}\right)$and hematopoietic $\left(\mathrm{CD} 45^{+}\right)$ stem cell populations. Given these reports, the most prudent path forward for an autologous cellular supplement to MG would be to evaluate serial depletions of the BMNC population. Furthermore, fractionation would allow the assessment of both of these myogenic stem cell populations without the added complication of the immune cell populations which might drive a fibrotic rather than myogenic response [21,22]. While not as clear cut as using freshly isolated BMNCs from a regulatory standpoint, the approach of using a fractionated autologous BMNC subpopulation is promising due to the maturity of immunomagentic separation as a clinical bioprocess [23-28].

A second hurdle to efficacy for this approach may reside in the delivery of a clinically impactful dose of BMNC-derived stem cells. This study focused on a single therapeutic dose of BMNCs (5 million cells per construct) freshly isolated via density gradient centrifugation, which did not result in an improvement in the 
functional capacity of the treated TA muscle relative to MG treatment alone. This BMNC dose and isolation methodology were chosen based on harvesting limitations of a single donor and the desire to utilize standard point of-care methodologies with a emphasis on clinical practicability. This dose, however, may vary in stem cell composition due to heterogeneity of both cell phenotype and composition. Furthermore, there is conflicting literature about the impact of density gradient centrifugation, and particularly the use of Ficoll as a medium, as an isolation methodology on the yield and potency of the resultant BMNC population [17, 29]. As such, these factors represent opportunities for optimization once depletion studies have elicited a candidate BMNC subpopulation for enrichment. It is possible that the optimal dose of BMNC-derived stem cells necessary to achieve meaningful gains in functional capacity may be superphysiological. This would necessitate an efficient isolation and ex vivo expansion methodologies to maximize yield of the effector stem cell populations or require a shift from an autologous to an allogenic approach. Both approaches face their own challenges, as ex vivo expansion raises additional manufacturing and regulatory challenges, while allogenic sourcing raises the possibility of originating graft-versus-host disease. As such, translation of either of these approaches would require further efforts to ensure their safety and feasibility. The benefit of a successful cellular supplement to MG therapy for VML would certainly justify further research into these approaches.

\section{Conclusions}

MG represents a promising solution to VML, a clinical problem with no current standard of care. MG, however, is reliant on sufficient autologous tissue to source the grafts. If this hurdle to clinical practice is to be overcome, supplemental therapies such as the concomitant delivery of BMNCs to the affected musculature will become necessary to achieve restoration of functional muscle tissue. While the results reported herein remain suboptimal, they present a promising treatment paradigm that is ripe for investigation and further development. Success of this paradigm will likely require meticulous lineage depletion studies to identify which BMNC subpopulations to include in an adjunct therapy and at what relative magnitude with respect to a given therapeutic MG dose. If this success is achieved, it is likely to have a significant clinical impact on the treatment of traumatic orthopedic wounds.

\section{Abbreviations}

BMNC: Bone marrow mononuclear cell; EDL: Extensor digitorum longus; GAPDH: Glyceraldehyde-3-phosphate dehydrogenase; GFP: Green fluorescent protein; MG: Minced muscle graft; RT-qPCR: Reverse transcriptase quantitative polymerase chain reaction; TA: Tibialis anterior; VML: Volumetric muscle loss

\section{Acknowledgements}

We thank Mr. Javier Chapa, Ms. Monica Farley, Ms. Monica Jalomo, Dr. Lisa Ji, and Ms. Shayna Levine for their technical assistance.

\section{Funding}

Funding was provided by the United States Army Medical Research and Materiel Command, Clinical and Rehabilitative Medical Research Program (Award\# C_003_2015_USAISR to BTC).

Availability of data and materials

All data generated or analyzed during this study are included in this published article.

\section{Authors' contributions}

SMG and BTC designed the experiments; SMG, BEPH, and BTC performed animal procedures; SMG and BEPH collected data; SMG and BTC analyzed results; SMG and BTC wrote and edited the manuscript. All authors approved the final version of the manuscript.

\section{Competing interests}

The opinions or assertions contained herein are the private views of the authors and are not to be construed as official or as reflecting the views of the Department of the Army or the Department of Defense. The authors declare that they have no competing interests.

\section{Consent for publication}

Not applicable.

\section{Ethics approval}

This study was conducted in compliance with the Animal Welfare Act, the implementing Animal Welfare Regulations and in accordance with the principles of the Guide for the Care and Use of Laboratory Animals. All animal procedures were approved by the US Army Institute of Surgical Research Institutional Animal Care and Use Committee.

\section{Publisher's Note}

Springer Nature remains neutral with regard to jurisdictional claims in published maps and institutional affiliations.

Received: 2 March 2017 Revised: 26 April 2017

Accepted: 17 May 2017 Published online: 09 June 2017

References

1. Grogan BF, Hsu JR. Volumetric muscle loss. J Am Acad Orthop Surg. 2011;19 Suppl 1:S35-7.

2. Corona BT, Garg K, Ward CL, MCDaniel JS, Walters TJ, Rathbone CR. Autologous minced muscle grafts: a tissue engineering therapy for the volumetric loss of skeletal muscle. Am J Physiol Cell Physiol. 2013;305(7):C761-75.

3. Ward CL, Ji L, Corona BT. An autologous muscle tissue expansion approach for the treatment of volumetric muscle loss. BioRes Open Access. 2015:4(1):198-208

4. Ward CL, Pollot BE, Goldman SM, Greising SM, Wenke JC, Corona BT. Autologous minced muscle grafts improve muscle strength in a porcine model of volumetric muscle loss injury. J Orthop Trauma. 2016;30(12):e396-403

5. Lepper C, Partridge TA, Fan CM. An absolute requirement for Pax7-positive satellite cells in acute injury-induced skeletal muscle regeneration. Development. 2011:138(17):3639-46.

6. Rouger K, Larcher T, Dubreil L, Deschamps J-Y, Le Guiner C, Jouvion G, Delorme B, Lieubeau B, Carlus M, Fornasari B, et al. Systemic delivery of allogenic muscle stem cells induces long-term muscle repair and clinical efficacy in Duchenne muscular dystrophy dogs. Am J Pathol. 2011;179(5):2501-18.

7. Minasi MG, Riminucci M, De Angelis L, Borello U, Berarducci B, Innocenzi A, Caprioli A, Sirabella D, Baiocchi M, De Maria R, et al. The mesoangioblast: a multipotent, self-renewing cell that originates from the dorsal aorta and differentiates into most mesodermal tissues. Development. 2002;129(11):2773-83.

8. Sherwood RI, Christensen JL, Conboy IM, Conboy MJ, Rando TA Weissman IL, Wagers AJ. Isolation of adult mouse myogenic 
progenitors: functional heterogeneity of cells within and engrafting skeletal muscle. Cell. 2004;119(4):543-54.

9. Polesskaya A, Seale P, Rudnicki MA. Wnt signaling induces the myogenic specification of resident CD45+ adult stem cells during muscle regeneration. Cell. 2003;113(7):841-52.

10. Cuende N, Rico L, Herrera C. Concise review: bone marrow mononuclear cells for the treatment of ischemic syndromes: medicinal product or cell transplantation? Stem Cells Transl Med. 2012;1(5):403-8.

11. Takahashi M, Li TS, Suzuki R, Kobayashi T, Ito H, Ikeda Y, Matsuzaki M, Hamano K. Cytokines produced by bone marrow cells can contribute to functional improvement of the infarcted heart by protecting cardiomyocytes from ischemic injury. Am J Physiol Heart Circ Physiol. 2006;291 (2):H886-93.

12. Ferrari G, Cusella-De Angelis G, Coletta M, Paolucci E, Stornaiuolo A, Cossu G, Mavilio F. Muscle regeneration by bone marrow-derived myogenic progenitors. Science. 1998;279(5356):1528-30.

13. Meyer S, Yarom R. Muscle regeneration and transplantation enhanced by bone marrow cells. Br J Exp Pathol. 1983;64(1):15-24.

14. Yarom R, Havivi Y. Acceleration of muscle regeneration by bone marrow cells. Experientia. 1977;33(2):195-7.

15. Yarom R, Meyer S, Carmy O, Ghidoni B, More R. Enhancement of human muscle growth in diffusion chambers by bone marrow cells. Virchows Arch B Cell Pathol Incl Mol Pathol. 1982;41(1-2):171-80.

16. Meyer $\mathrm{S}$, Kenan $\mathrm{S}$, Yarom R. Enhancement of muscle regeneration by bone marrow cells in the monkey. Experientia. 1984;40(5):490-2.

17. Pösel C, Möller K, Fröhlich W, Schulz I, Boltze J, Wagner D-C. Density gradient centrifugation compromises bone marrow mononuclear cell yield. PLoS One. 2012;7(12):e50293.

18. Assmus B, Tonn T, Seeger FH, Yoon CH, Leistner D, Klotsche J, Schachinger $V$, Seifried E, Zeiher AM, Dimmeler $S$. Red blood cell contamination of the final cell product impairs the efficacy of autologous bone marrow mononuclear cell therapy. J Am Coll Cardiol. 2010;55(13):1385-94.

19. Sharma A, Sane H, Paranjape A, Bhagawanani K, Gokulchandran N, Badhe P. Autologous bone marrow mononuclear cell transplantation in Duchenne muscular dystrophy —a case report. Am J Case Rep. 2014;15:128-34.

20. Sharma A, Kulkarnia P, Chopra G, Gokulchandran N, Lohia M, Badhe P. Autologous bone marrow-derived mononuclear cell transplantation in Duchenne muscular dystrophy. Ind J Clin Pract. 2012;23(3):169-72.

21. Serrano AL, Munoz-Canoves $P$. Regulation and dysregulation of fibrosis in skeletal muscle. Exp Cell Res. 2010;316(18):3050-8.

22. Mann CJ, Perdiquero E, Kharraz Y, Aguilar S, Pessina P, Serrano AL, Muñoz-Cánoves P. Aberrant repair and fibrosis development in skeletal muscle. Skelet Muscle. 2011;1(1):21.

23. Pasquini MC, Devine S, Mendizabal A, Baden LR, Wingard JR, Lazarus HM, Appelbaum FR, Keever-Taylor CA, Horowitz MM, Carter S, et al. Comparative outcomes of donor graft CD34+ selection and immune suppressive therapy as graft-versus-host disease prophylaxis for patients with acute myeloid leukemia in complete remission undergoing HLA-matched sibling allogeneic hematopoietic cell transplantation. J Clin Oncol. 2012;30(26):3194-201

24. Barfield RC, Otto M, Houston J, Holladay M, Geiger T, Martin J, Leimig T, Gordon P, Chen X, Handgretinger R. A one-step large-scale method for T- and B-cell depletion of mobilized PBSC for allogeneic transplantation. Cytotherapy. 2004;6(1):1-6.

25. Bornhauser M, Platzbecker U, Theuser C, Holig K, Ehninger G. CD34 + -enriched peripheral blood progenitor cells from unrelated donors for allografting of adult patients: high risk of graft failure, infection and relapse despite donor lymphocyte add-back. Br J Haematol. 2002;118(4):1095-103.

26. Elmaagacli AH, Peceny R, Steckel N, Trenschel R, Ottinger $H$, Grosse-Wilde $H$, Schaefer UW, Beelen DW. Outcome of transplantation of highly purified peripheral blood CD34+ cells with T-cell add-back compared with unmanipulated bone marrow or peripheral blood stem cells from HLAidentical sibling donors in patients with first chronic phase chronic myeloid leukemia. Blood. 2003;101(2):446-53.

27. Handgretinger R, Klingebiel T, Lang P, Schumm M, Neu S, Geiselhart A, Bader P, Schlegel PG, Greil J, Stachel D, et al. Megadose transplantation of purified peripheral blood CD34(+) progenitor cells from HLA-mismatched parental donors in children. Bone Marrow Transplant. 2001;27(8):777-83.

28. Aversa F, Terenzi A, Tabilio A, Falzetti F, Carotti A, Ballanti S, Felicini R, Falcinelli F, Velardi A, Ruggeri L, et al. Full haplotype-mismatched hematopoietic stem-cell transplantation: a phase II study in patients with acute leukemia at high risk of relapse. J Clin Oncol. 2005;23(15):3447-54.
29. Hernández P, Cortina L, Artaza H, Pol N, Lam R, Dorticós E, Macías C, Hernández C, Del Valle L, Blanco A. Autologous bone-marrow mononuclear cell implantation in patients with severe lower limb ischaemia: a comparison of using blood cell separator and Ficoll density gradient centrifugation. Atherosclerosis. 2007;194(2):e52-6.

\section{Submit your next manuscript to BioMed Central and we will help you at every step:}

- We accept pre-submission inquiries

- Our selector tool helps you to find the most relevant journal

- We provide round the clock customer support

- Convenient online submission

- Thorough peer review

- Inclusion in PubMed and all major indexing services

- Maximum visibility for your research

Submit your manuscript at www.biomedcentral.com/submit
Biomed Central 